\title{
Design and optimization of cascaded DCG based holographic elements for spectrum-splitting PV systems
}

Yuechen Wu, Benjamin Chrysler, Silvana Ayala Pelaez, Raymond K. Kostuk

Yuechen Wu, Benjamin Chrysler, Silvana Ayala Pelaez, Raymond K. Kostuk, "Design and optimization of cascaded DCG based holographic elements for spectrum-splitting PV systems," Proc. SPIE 10379, Nonimaging Optics: Efficient Design for Illumination and Solar Concentration XIV, 103790T (17 October 2017); doi: 10.1117/12.2273229

SPIE Event: SPIE Optical Engineering + Applications, 2017, San Diego, California, United States 


\title{
Design and optimization of cascaded DCG based holographic elements for spectrum-splitting PV systems
}

\author{
Yuechen $\mathrm{Wu}^{\mathrm{a}}$, Benjamin Chrysler ${ }^{\mathrm{b}}$, Silvana Ayala Palaez ${ }^{\mathrm{a}}$, Raymond K. Kostuk ${ }^{\mathrm{a}, \mathrm{b}}$
}

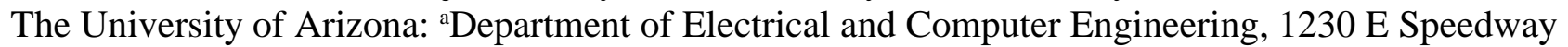

Blvd. Tucson AZ 85721; 'College of Optical Sciences, 1630 E. University Blvd. Tucson, AZ 85721

\begin{abstract}
In this work, the technique of designing and optimizing broadband volume transmission holograms using dichromate gelatin (DCG) is summarized for solar spectrum-splitting application. Spectrum splitting photovoltaic system uses a series of single bandgap PV cells that have different spectral conversion efficiency properties to more fully utilize the solar spectrum. In such a system, one or more high performance optical filters are usually required to split the solar spectrum and efficiently send them to the corresponding PV cells. An ideal spectral filter should have a rectangular shape with sharp transition wavelengths. DCG is a near ideal holographic material for solar applications as it can achieve high refractive index modulation, low absorption and scattering properties and long-term stability to solar exposure after sealing. In this research, a methodology of designing and modeling a transmission DCG hologram using coupled wave analysis for different PV bandgap combinations is described. To achieve a broad diffraction bandwidth and sharp cut-off wavelength, a cascaded structure of multiple thick holograms is described. A search algorithm is also developed to optimize both single and two-layer cascaded holographic spectrum splitters for the best bandgap combinations of twoand three-junction SSPV systems illuminated under the AM1.5 solar spectrum. The power conversion efficiencies of the optimized systems under the AM1.5 solar spectrum are then calculated using the detailed balance method, and shows an improvement compared with tandem structure.
\end{abstract}

Key worlds: Solar energy, Spectrum splitting, Multi-junction PV, Holography, Volume hologram

\section{INTRODUCTION}

In order to make better use of the power available in the complete solar spectrum, photovoltaic (PV) cells with several energy bandgaps are required ${ }^{1-2}$. This approach allows the efficiency to exceed the detailed balance limit for single bandgap systems of $33 \%^{3}$. Two approaches to multi-junction photovoltaic (MJPV) system design have been developed. In the most common method a broadband concentrator (BBC) is used to collect light and focus it onto the surface of a tandem MJPV cell. This method allows for high optical efficiencies and power conversion efficiencies in excess of 45\%. The complexity of BBC-MJPV systems is in the tandem MJPV cell which consists of different energy bandgap materials grown on top of one another. This requires precise lattice matching between layers. In addition, the current output of the complete cell is limited by the lowest output junction. Tandem MJPV cells are expensive to fabricate and therefore high concentration ratios are used to limit the size of the cell ${ }^{4}$. This in turn restricts the field of view (FOV) of the collector and requires direct sunlight and high accuracy tracking for optimal performance.

An alternative to BBC-MJPV cell systems are spectrum splitting collectors (SSC) with a set of single junction PV (SJPV) cells that have different energy bandgaps ${ }^{5-10}$. In this approach the optical system spatially separates incident solar illumination into different spectral bands and directs each band to a PV cell that has the highest response to the spectral band. Since each PV cell is separated lattice matching is not an issue and different material types can be used ${ }^{11}$. In addition, the cells are connected in parallel and the total current is not current limited by the lowest output cell ${ }^{12}$. However, the complexity of the system resides in the optics which must effectively separate the different spectral bands of the incident solar illumination and direct them to corresponding cells. The optics must also perform a certain level of concentration at the PV cell surface ${ }^{13}$.

In this paper, a methodology of designing and modeling a transmission DCG holograms for a dispersive spectrum splitting concentrating PV system using coupled wave analysis is described. To achieve a broad spectral bandwidth with sharp cut-off wavelength, a cascaded structure of multiple thick holograms is evaluated and compared with single layer holographic spectrum splitting elements. A search algorithm is also developed to optimize both single layer and two-layer cascaded holographic spectrum splitting elements for the best mathch with two- and three-junction SSPV systems illuminated under AM1.5 solar spectrum. A detailed balance analysis method is then used to determine the maximum limit of power conversion efficiency under AM1.5 solar illumination for the two- and threejunction dispersed SSCPV system.

*ywu2@email.arizona.edu

Nonimaging Optics: Efficient Design for Illumination and Solar Concentration XIV,

edited by Roland Winston, Sarah R. Kurtz, Proc. of SPIE Vol. 10379, 103790T

(c) 2017 SPIE - CCC code: 0277-786X/17/\$18 - doi: 10.1117/12.2273229

Proc. of SPIE Vol. 10379 103790T-1 


\section{EVALUATING SPECTRUM SPLITTING SYSTEM}

Spectrum splitting optics must spatially separate different spectral bands of incident solar illumination and direct each band to a specific PV cell. This allows dissimilar PV materials to be used and is not restricted by current matching effects Optical spectrum splitting can effectively be achieved with reflective non-dispersive and dispersive transmission configurations. In the reflection geometry one or more reflection band pass filters are used that reflect the desired spectral band into a PV cell; and transmits the rest into the other PV cell or the next filter (Figure 1) ${ }^{9}$. The dispersion approach applies a transmitted dispersive optics which could be either prisms or diffraction gratings to laterally split the incident spectrum at a receiver plan $^{5-8}$. Compared with reflection approach, the dispersive spectrum splitting benefits from its simple structure of a system with more than two junctions, and the ability of mass production. However, to achieve a high efficient dispersive SSPV system, a filter that can efficiently and accurately send the photons into the right location at the receiver plane are required.
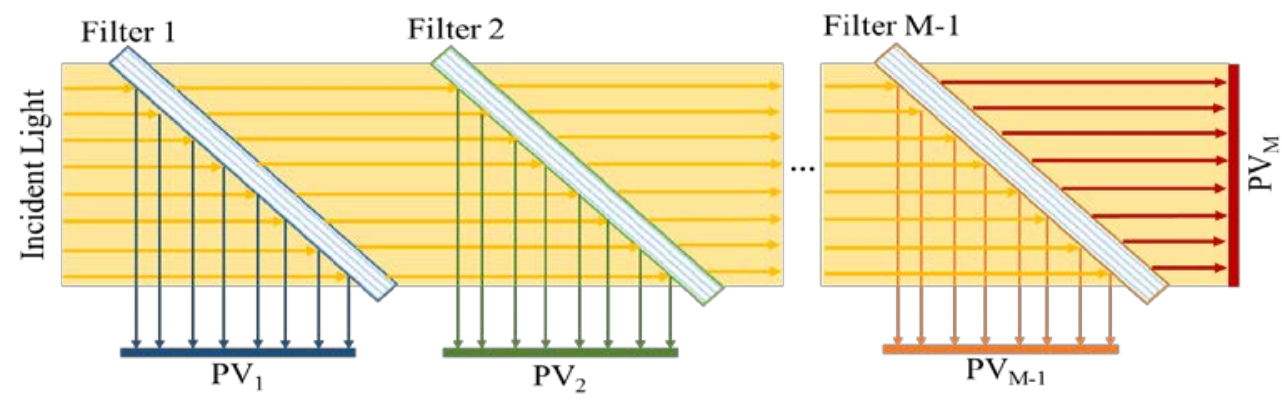

Figure 1. Spectrum splitting PV system using reflective type filters.

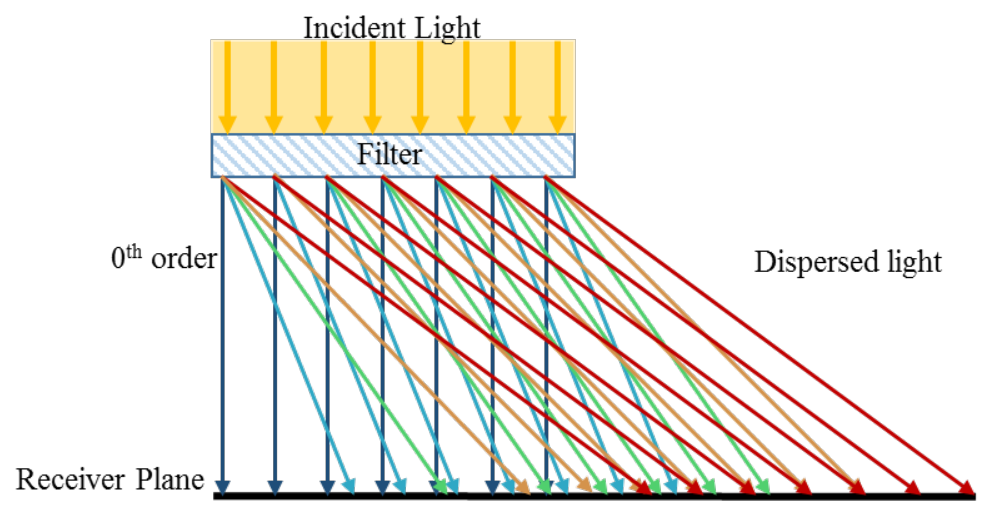

(a)

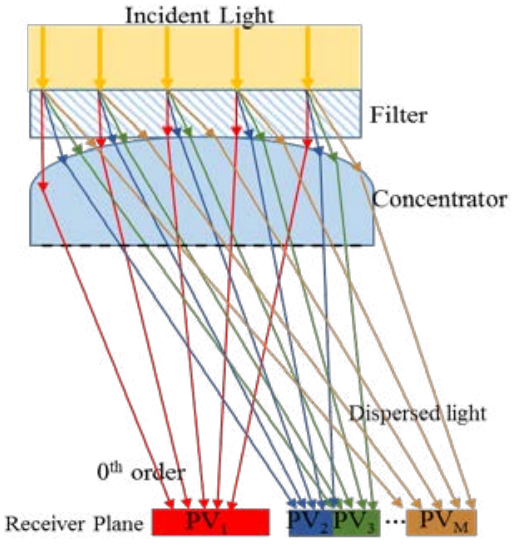

(b)

Figure 2. Dispersive spectrum splitting PV system. (a) A single dispersive filter projects the complete spectrum on the receiver plane. Wavelengths overlaps to each other at the receiver plane. (b) A lens is combined with the dispersive filter to focus light and minimize the overlap between spectral bands.

\subsection{Efficiency of SSPV systems}

The spectrum splitting element in a SSPV system matches the PV cell spectral responsivity with portions of the incident spectrum to achieve higher conversion efficiency. In an N-junction SSPV system, the power conversion efficiency of the $\mathrm{k}^{\text {th }}$ solar cell can be expressed as:

$$
\eta_{k}=\frac{1}{P} \int_{-\infty}^{+\infty} E(\lambda) \eta_{k}^{o p t}(\lambda) S C E_{k}(\lambda) d \lambda,
$$

where, $\eta_{k}$ is the filtered power conversion efficiency of the $\mathrm{k}^{\text {th }}$ solar cell; $\mathrm{E}(\lambda)$ is the incident power spectrum; $\mathrm{P}$ is the total incident power; $\eta_{k}^{\text {opt }}(\lambda)$ is the spectral optical efficiency of the optical filter for the wavelengths corresponding to the kth cell; and the $S C E_{k}(\lambda)$ is the spectrum conversion efficiency (SCE) of the kth cell. An accurate expression for $S C E_{k}(\lambda)$ can be calculated using the detailed balance method with the incident power spectrum ${ }^{6}$. 
The overall power conversion efficiency for such a system can then be calculated with:

$$
\eta_{s y s}=\sum_{k=1}^{N} \eta_{k}
$$

The goal of optimizing a SSPV system is to maximize the optical power conversion efficiency with the incident solar spectral power distribution. This requires that: 1) each PV cells in the system either increases the SCE over other cells in regions where the spectral responsivity overlaps or extends the wavelength range of the solar spectrum that is converted; 2) the optical efficiency of the filter $\left(\eta_{k}^{o p t}(\lambda)\right)$ is maximized within its spectral band, and minimized elsewhere. In this paper, optical filter properties are evaluated and optimized for spectrum splitting PV (SSPV) systems.

\subsection{Evaluating optical filters}

In a spectrum splitting system the product of the spectral responsivity of the PV cells and the filter function contribute to the overall efficiency of the system. The properties of an ideal filter for a particular PV cell (k) can be expressed as:

$$
\eta_{k, \text { ideal }}^{\text {opt }}(\lambda)=\left\{\begin{array}{lr}
1 & \left(\frac{h c}{E_{g}^{k-1}} \leq \lambda<\frac{h c}{E_{g}^{k}}\right) \\
0 & \text { Otherwise }
\end{array},\right.
$$

where $\mathrm{h}$ is the Planck constant, c is the speed of light, $E_{g}^{k}$ is the bandgap energy of the $\mathrm{k}^{\text {th }} \mathrm{PV}$ cell, and $E_{g}^{k}$ is the bandgap energy of the $(k-1)^{\text {th }}$ PV cell that has higher bandgap energy than the $k^{\text {th }}$ PV cell. In actual systems, the filter efficiency will be less than 1 for at least part of the spectral band and reduces the conversion efficiency of the SSPV system.

To evaluate only the performance of the optical filters, we assume that the PV cells using in the system absorbs all the photons with energy less than their bandgap energy (i.e. EQE=1). Then Eq. (1) can be simplified to be:

$$
\begin{aligned}
\eta_{k} & =\frac{1}{P} \int_{-\infty}^{+\infty} E(\lambda) \eta_{k}^{o p t}(\lambda) S C E_{k}(\lambda) d \lambda \\
& =\frac{1}{P} \int_{-\infty}^{+\infty} Q(\lambda)\left(\frac{h c}{\lambda}\right) \eta_{k}^{o p t}(\lambda) \frac{E Q E_{k}(\lambda)}{h c / \lambda} q V_{o c} F F d \lambda \\
& =\frac{1}{P} \int_{-\infty}^{+\infty} Q(\lambda) \eta_{k}^{o p t}(\lambda)\left[E Q E_{k}(\lambda) q V_{o c} F F\right] d \lambda \\
& =C\left(E_{g}^{k}\right) \int_{\lambda_{1}}^{\lambda_{2}} Q(\lambda) \eta_{k}^{o p t}(\lambda) d \lambda ;
\end{aligned}
$$

where $Q(\lambda)$ is the total number of incident photons at wavelength $\lambda, \lambda_{1}$ and $\lambda_{2}$ are the wavelengths that have photon energies equal to the bandgap energies of the $\mathrm{k}^{\text {th }}$ and $(\mathrm{k}-1)^{\text {th }} \mathrm{PV}$ cells, $E Q E_{k}(\lambda)$ is the external quantum efficiency of the $\mathrm{k}^{\text {th }} \mathrm{PV}$ cell, which is equal to 1 when $\lambda$ is with in $\left[\lambda_{1}, \lambda_{2}\right], \mathrm{hc} / \lambda$ is the photon energy of the photon with wavelength $\lambda$, and $\mathrm{q}$ is the electron charge. Voc and FF are the open circuit voltage and fill factor of the PV cell which can be defined using detail balance analysis. To simplify the analysis, Voc and FF are assumed to be independent on the wavelength. So, $C\left(E_{g}^{k}\right)$ is a constant that only related to the bandgap energy of the $\mathrm{k}^{\text {th }} \mathrm{PV}$ cell.

Therefore, to optimize Eq. (4), the practical optical filters using in SSPV systems functioning under a certain illumination condition can be evaluated with an ideal factor. It is defined as the average of weighted optical quantum efficiencies over the desired filtering spectral band:

$$
\text { Ideal Factor }{ }_{k}=\frac{1}{Q_{\lambda_{1}, \lambda_{2}}} \int_{\lambda_{1}}^{\lambda_{2}} \eta_{k}^{o p t}(\lambda) \times Q(\lambda) d \lambda,
$$

where $n_{k}$ is the ideal factor of the filtering optics of the $\mathrm{k}^{\text {th }} \mathrm{PV}$ cell, $Q_{\lambda_{1}, \lambda_{2}}$ is the total number of incident photons within the desired filtering spectral band: $\left[\lambda_{1}, \lambda_{2}\right]$. This ideal factor compares the number of photons that arrive and are absorbed by the kth PV cell with the total number of incident photons within the desired absorption spectral band, because the maximum power output of a PV cell is positive correlated to the number of photons that are absorbed (i.e. the number of electric-hole pairs been generated).

However, in an optical system, there are interconnection between Ideal Factor ${ }_{k}$ and Ieal Factor I $_{k+1}$ as shown in Figure.3. So, to optimize the entire system, it is necessary to consider an overall optical ideal factor that includes all involved spectral band and the contribution of each bandgap. This system optical factor is then defined as: 


$$
\text { Optical Factor }=\frac{\sum_{k=1}^{N} n_{k} \times E_{g}^{k}}{\sum_{k=1}^{N} E_{g}^{k}},
$$

Then, this system optical factor will be used as the figure of merit in spectrum splitter optimization.

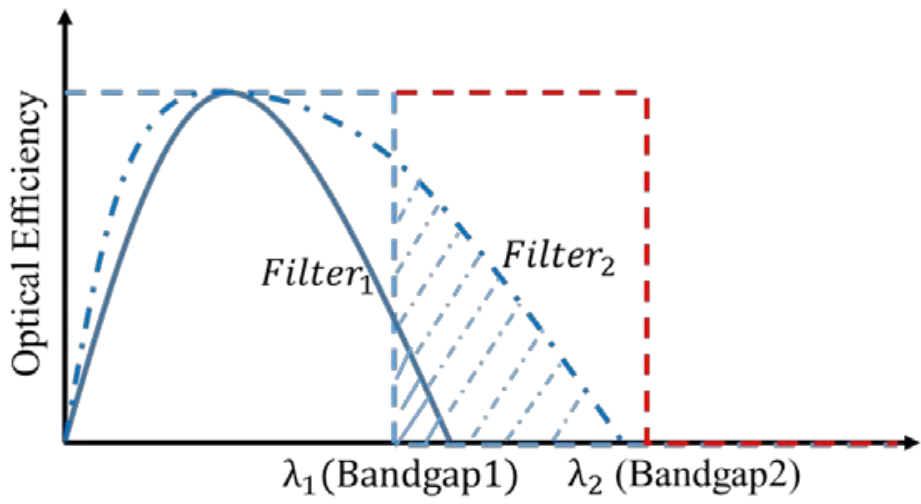

Figure 3. Interconnection between ideal factors. Filter2 has a larger ideal factor than filter 1 for bandgap1 PV cells. However, it also results a lower ideal factor than Filter 1 for bandgap2.

In a dispersive SSPV system, the loss resulting from the optical elements is usually due to: 1) mismatch between diffracted spectrum and the desired absorption band of the PV cell; 2) low diffraction efficiency over the spectral band diffracted by the hologram; 3) overlap between diffracted spectral bands. Several papers have demonstrated that the losses from 3) can be minimized by concentrating the dispersed spectral bands diffracted by the holographic elements. This can be achieved either by using an additional focusing system ${ }^{5-7}$, or adding power directly into the diffractive element ${ }^{8}$. In this research, we only focused on minimizing the loss from 1) and 2) of the holographic spectrum splitting elements, and assume no loss due to 3). The fundamental power conversion efficiency limits are then found for 2- and 3-junction SSPV systems using single layer and cascaded structured holographic spectrum splitting elements.

\subsection{Bandgap combinations}

The bandgap combination for the SSPV system is determined to obtain the maximum power conversion efficiency of the system when Ideal Factor ${ }_{k}=1$ for all bandgaps. Under this condition, the method of calculating fundamental detailed balance limitation of N-junction PV system under AM1.5G spectrum has been addressed by S.P. Bremner in $2007^{15}$. By following this method, the optimized bandgap combinations and the efficiency limitations for 2-, 3-, and 4-junction systems without current match constraints have been found and listed in Table 1. These combinations will then be applied to a sample SSCPV system and used to optimize the holographic spectrum splitting elements.

Table 1. The optimized sets of bandgaps and corresponding transition wavelengths for 2-, 3-, and 4-junction MJPV system in standard one sun condition

\begin{tabular}{|c|c|c|c|c|c|c|c|c|c|}
\hline $\mathbf{N}$ & $\boldsymbol{\eta} \mathbf{( \% )}$ & $\mathbf{E}_{\mathbf{g} 1}(\mathbf{e V})$ & $\mathbf{E}_{\mathbf{g} 2}(\mathbf{e V})$ & $\mathbf{E}_{\mathbf{g} 3}(\mathbf{e V})$ & $\mathbf{E}_{\mathbf{g} 4}(\mathbf{e V})$ & $\boldsymbol{\lambda}_{\mathbf{1}} \mathbf{( n m )}$ & $\boldsymbol{\lambda}_{\mathbf{2}} \mathbf{( n m )}$ & $\boldsymbol{\lambda}_{\mathbf{3}} \mathbf{( n m )}$ & $\boldsymbol{\lambda}_{\mathbf{4}} \mathbf{( n m )}$ \\
\hline 2 & 46.06 & 1.73 & 0.94 & - & - & 717 & 1319 & - & - \\
\hline 3 & 51.94 & 2.05 & 1.40 & 0.93 & - & 605 & 886 & 1333 & - \\
\hline 4 & 55.91 & 2.23 & 1.64 & 1.13 & 0.70 & 556 & 756 & 1097 & 1771 \\
\hline
\end{tabular}

\section{HOLOGRAPHIC SPECTRUM SPLITTING ELEMENTS}

Volume holographic elements are a good for spectrum splitting elements used in SSPV systems, especially for dispersive transmission designs. This is due to the fact that volume holograms can be designed to diffract a specific spectral band with high diffraction efficiency (DE) and is dispersive due to its grating properties. The elements can also be mass produced at low cost. Dichromated gelatin (DCG) is a well-known material for making high quality volume holograms ${ }^{14}$. This material can provide a wide range of refractive index modulation (0.001 to 0.1 ) and has long-term stability properties under 
solar illumination after being sealed ${ }^{16}$. With using different deposition process, the film thickness can also be controlled from $2 \mu \mathrm{m}$ to $50 \mu \mathrm{m}$ to control the initial spectral bandwidth range. Further modification to the shape and width of the diffracted spectral bandwidth can be achieved by varying the film processing method.

In this research, we focused on optimizing DCG based holographic spectrum splitting elements to obtain the most efficient 2- and 3- junction SSPV systems (Table 1.). The optimization process focuses on maximizing the system optical factor shown in Eq. (6). For each of the corresponding bandgap combinations shown in table 1., both single and two-layer cascaded holographic spectrum splitting elements are designed and optimized. The power conversion efficiencies for the corresponding systems are then calculated using detailed balance method to obtain the limits of SSPV systems using holographic spectrum splitting elements.

\subsection{SSPV system model}

A unit element of the holographic dispersive spectrum splitting system is illustrated in Figure 4.. This system consists of a holographic filter placed above an aspherical lens array to direct different components of solar spectrum onto the most efficient PV cells. A series of PV cells with different bandgap energies are arranged horizontally at the receiver plane to receive the corresponding spectrum components. The narrow bandgap PV cell is located along the axis of the system and the wider bandgap cells are located at a position corresponding to the designed diffraction angle. The hologram is designed to diffract normally incident light. This allows diffuse light entering at large angles to be converted by the narrow bandgap PV cell to increase energy yield.

In this study, we aimed at finding the efficiency limit of a DCG based holographic SSPV system. A few assumptions are made to simplify the model:

1) The concentrating lens is perfect: no aberrations or residual dispersion by the lens.

2) The concentration ratio is high enough to avoid spectral overlap at the PV cells.

3) PV cells used in the system absorb all photons that exceed the energy bandgap. Detailed balance method is used to calculate the power conversion efficiency of each cells in the system.

4) The holographic spectrum splitting elements satisfy the conditions for approximate coupled wave analysis ${ }^{16}$.

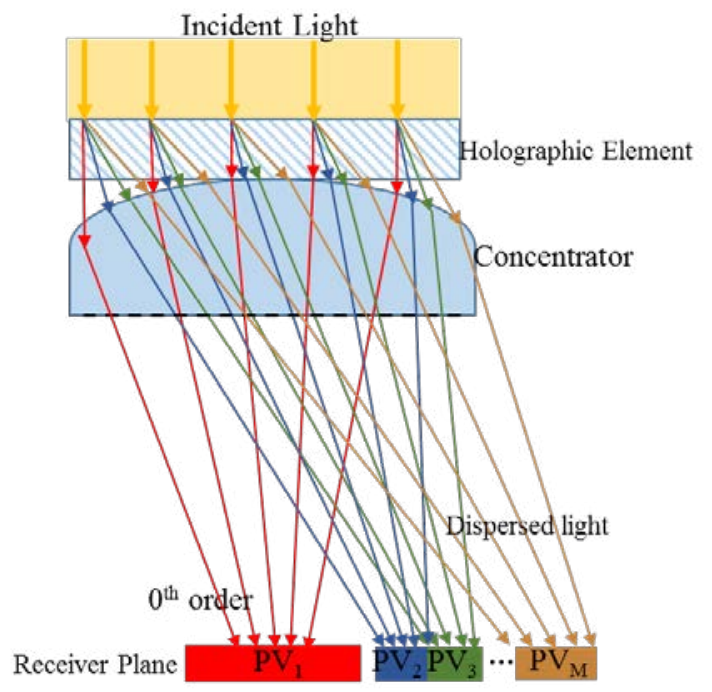

Figure 4. The structure of a dispersive holographic spectrum splitting system with a perfect focusing element.

\subsection{Modeling holographic spectrum splitter}

The holographic gratings using in this study are modeled with Kogelink's coupled wave analysis ${ }^{17}$. The variables used in optimization includ the hologram thickness, refrective index modulation, grating period, peak diffraction wavelength, and 
diffraction angles. The hologram parameters are consistent with the capability of the DCG material and the design of the holographic SSPV system.

\subsubsection{Single layer spectrum splitting elements}

The single layer holographic spectrum splitting elements are designed and optimized based on the Kogelink's coupled wave model and the figure of merit defined in section 2.2 for both 2- and 3- junction SSPV systems. The design considerations include: 1) the diffraction angle the wavelength that determines the surface grating period (x component of the grating vector: $\overrightarrow{\mathrm{K}}$ ); 2 ) the wavelength that optimizes the diffraction efficiency to maximize the conversion efficiency of the PV cells. This wavelength is Bragg matched to the volume grating vector by determining z component of the $\overrightarrow{\mathrm{K}}$ vector. 3) The spectral diffraction efficiency and bandwidth, which are determined by the hologram material properties including the index modulation, film thickness, and $\overrightarrow{\mathrm{K}}$ vector.

The first step to designing a transmission volume hologram is to determine the grating periods in both surface and depth direction. The filters are designed for normal incident illumination. To obtain high diffraction efficiency at the desired peak efficiency wavelength with a certain diffraction angle, both the grating equation and Bragg condition ${ }^{16}$ must be satisfied. In this case the grating period components along the surface direction, $\Lambda_{x}$, and depth direction, $\Lambda_{z}$, can then be determined according to:

$$
\begin{aligned}
& \Lambda_{x}=\frac{\lambda_{\text {Bragg }}}{n \sin \left(\theta_{\text {Bragg }}\right)}, \\
& \Lambda_{z}=\frac{\lambda_{\text {Bragg }}}{n\left[1-\cos \left(\theta_{\text {Bragg }}\right)\right]},
\end{aligned}
$$

Where $\lambda_{B r a g g}$ is the Bragg matched wavelength, $\mathrm{n}$ is the average refractive index of the hologram material, and $\theta_{B r a g g}$ is the diffraction angle of $\lambda_{\text {Bragg }}$.

The next step is to use the Kogelink's approximate coupled wave analysis to calculate the spectral diffraction efficiency of the volume transmission phase grating:

$$
\eta(\lambda)=\frac{\sin ^{2}\left(v(\lambda)^{2}+\xi(\lambda)^{2}\right)^{1 / 2}}{\left(1+\xi(\lambda)^{2} / v(\lambda)^{2}\right)}
$$

where, $\eta(\lambda)$ is the spectral diffraction efficiency, $v(\lambda)=\frac{\pi n_{1} h}{\lambda c_{r}(\lambda) c_{s}(\lambda)}$ is the grating strength parameter, $\xi(\lambda)$ is a parameter defined as $\xi(\lambda)=\frac{\vartheta(\lambda) h}{2 c_{s}(\lambda)}, n_{1}$ is the refractive index modulation, $\mathrm{h}$ is the thickness of the grating, $c_{r}$ and $c_{s}$ are the cosine values of the incident and diffracted beams, and $\vartheta(\lambda)$ is the detuning parameter.

A final design condition for the holographic elements is to satisfy the volume quality or Q factor for the grating defined by $Q=\frac{2 \pi \lambda_{\text {peak }} h}{n \Lambda^{2}} \geq 10$. Having the hologram parameters satisfy this relation ensures that the volume condition is satisfy and that a single high efficiency diffraction order will result.

Table 2. Hologram optimization search parameter range and step increments.

\begin{tabular}{|l|c|c|c|c|}
\hline & \multicolumn{2}{|c|}{ Two-Junction (1.73eV; 0.94eV) } & Three-Junction (2.05eV; 1.4eV; 0.93eV) \\
\hline & Range (min-max) & Step Separation & Range (min-max) & Step Separation \\
\hline$\lambda_{\text {Bragg }}(\mathrm{nm})$ & $300-717$ & 5 & $300-886$ & 5 \\
\hline$\theta_{\text {Bragg }}$ (degree) & $10-25$ & 1 & $10-35$ & 1 \\
\hline Grating Thickness $(\mu \mathrm{m})$ & $1-30$ & 1 & $1-30$ & 0.002 \\
\hline Index Modulation & $0-0.1$ & 0.002 & $0-0.1$ & 1 \\
\hline
\end{tabular}

Based on this model, the spectral optical properties of a single layer spectrum splitting element can be modified by setting the: 1) Bragg matched wavelength; 2) diffraction angle of $\lambda_{\text {Bragg }}$; 3 ) grating thickness; and 4) refractive index modulation. 
To optimize a hologram for specific SSPV system, a search algorithm is used that searches through the possible values of these four parameters to obtain the highest overall optical factor for the desired 2- and 3- junction SSPV system. Table 2. shows the searching range and step increment for each parameter, and they are based on the possible DCG material properties.

\subsubsection{Two-layer cascaded spectrum splitting element}

The two-layer cascaded spectrum splitting element consists of two volume stacked holograms as shown in Figure 5. It is easier to achieve a `near ideal' filter function with the cascaded configuration due to a larger set of hologram design parameters. In general, it is possible to achieve both a broader spectral bandwidth and sharper transition edge for the resulting filter.

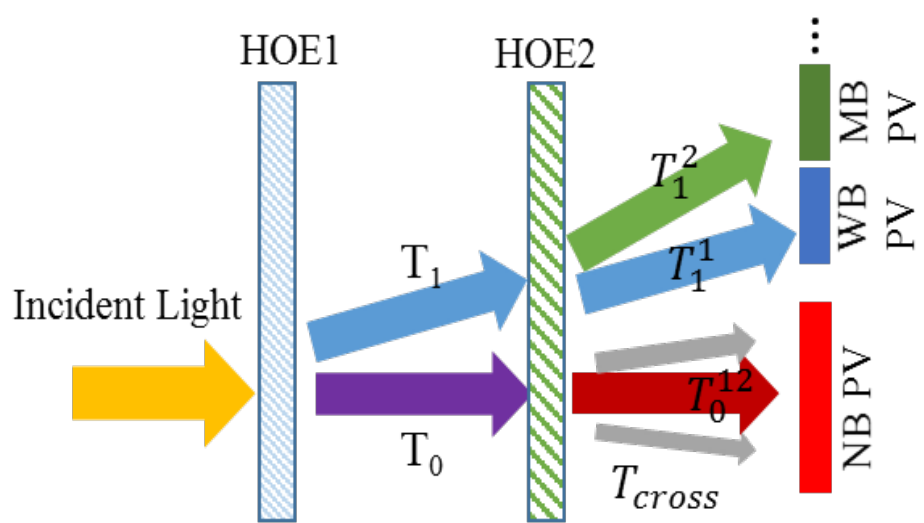

Figure 5. Input and output orders and PV cell locations for a two-layer cascaded SSPV system.

However, the holograms must be designed to minimize cross coupling effects. As shown in Figure 4., if the same diffraction angles and wavelengths are used for both gratings, light diffracted by HOE1 into the first diffracted order T1 will be coupled back into the zero order $\left(\mathrm{T}_{0}{ }^{12}\right)$. If cross-coupling is not minimized, the diffracted spectral components will not reach the PV cell for maximum conversion efficiency.

To optimize a two-layer cascaded holographic spectrum splitter, both the top hologram and the bottom hologram are optimized by searching through the parameters shown in Table 2 using single hologram model described in section 3.2.1. In each iteration, the spectral diffraction efficiencies of HOE1 and HOE2 $\left(\eta_{1}(\lambda)\right.$ and $\left.\eta_{2}(\lambda)\right)$, and the cross-coupling efficiency $\left(\eta_{\text {cross }}(\lambda)\right)$ for $\mathrm{T}_{1}$ passing through the HOE2 are calculated. Then the overall filtering efficiency for this grating can be expressed as:

$$
\eta(\lambda)=\eta_{1}(\lambda) \times\left(1-\eta_{\text {recouple }}(\lambda)\right)+\left(1-\eta_{1}(\lambda)\right) \times \eta_{2}(\lambda),
$$

Then, this efficiency is applied to the model of SSPV system to calculate the overall optical ideal factor. After searching all the parameters for HOE1 and HOE2, optimized Two-layer cascaded spectrum splitters are found for both 2- and 3junction SSPV systems.

\subsection{Optimization and modeling results}

Based on the volume grating model and the optimization methods described in section 3.2, programs are created and run to find the optimized holographic optical elements (HOEs) for the two- and three-junction SSPV systems that are described in section 3.1. All the optimized parameters for the HOEs are found for the best bandgap combinations $(1.73 \mathrm{eV}$ and $0.94 \mathrm{eV}$ for two-junction; $2.05 \mathrm{eV}, 1.4 \mathrm{eV}$, and $0.93 \mathrm{eV}$ for three-junction). These parameters are summarized in Table 3 . The results show that the optimized system optical factor for the two-junction SSPV system reaches 0.836 with single HOE and 0.895 with two-layer cascaded HOE, and for the three-junction system reaches 0.7941 with single HOE, and 0.8411 with the two-layer cascaded filter. 
Table 3. The optimized parameters for the single HOEs and the cascaded filters using in the optimized two- and three- junction spectrum splitting PV system.

\begin{tabular}{|c|c|c|c|c|c|c|}
\hline \multirow{3}{*}{ Optimized Parameters } & \multicolumn{3}{|c|}{ Two-Junction $(1.73 \mathrm{eV} ; 0.94 \mathrm{eV})$} & \multicolumn{3}{|c|}{ Three-Junction $(2.05 \mathrm{eV} ; 1.4 \mathrm{eV} ; 0.93 \mathrm{eV})$} \\
\hline & \multirow[t]{2}{*}{ Single HOE } & \multicolumn{2}{|c|}{ HOEs in Cascaded Filter } & \multirow[t]{2}{*}{ Single HOE } & \multicolumn{2}{|c|}{ HOEs in Cascaded Filter } \\
\hline & & Top (1) & Bottom (2) & & Top (1) & Bottom (2) \\
\hline Ideal Factor (WBF): & 0.8236 & \multicolumn{2}{|c|}{0.894} & 0.8431 & \multicolumn{2}{|c|}{0.8582} \\
\hline Ideal Factor (MBF): & - & \multicolumn{2}{|c|}{-} & 0.5813 & \multicolumn{2}{|c|}{0.756} \\
\hline Ideal Factor (NBF): & 0.8593 & \multicolumn{2}{|c|}{0.8981} & 0.947 & \multicolumn{2}{|c|}{0.9313} \\
\hline System Optical Factor: & 0.8361 & \multicolumn{2}{|c|}{0.8954} & 0.7941 & \multicolumn{2}{|c|}{0.8411} \\
\hline Bragg mached WL (nm): & 525 & 500 & 500 & 565 & 535 & 805 \\
\hline Diffraction Angle (degree): & 21 & 21 & 14 & 23 & 18 & 31 \\
\hline$\Lambda \mathrm{x}(\mu \mathrm{m})$ & 0.943 & 0.943 & 1.3508 & 0.943 & 1.1292 & 1.012 \\
\hline$\Lambda \mathrm{z}(\mu \mathrm{m})$ & 5.0063 & 5.2746 & 11.002 & 4.6247 & 7.1134 & 3.6094 \\
\hline Grating Thickness $(\mu \mathrm{m})$ : & 4 & 16 & 12 & 4 & 6 & 14 \\
\hline Index Modulation: & 0.06 & 0.09 & 0.02 & 0.064 & 0.042 & 0.022 \\
\hline Q Factor: & 10.0404 & 87.0526 & 41.4007 & 11.3906 & 10.5994 & 48.7424 \\
\hline
\end{tabular}

The spectral optical filtering efficiencies (SOFE) for the optimized two- and three- junction SSPV systems when illuminated with an AM1.5 illumination power spectrum (PS) are plotted in Figure 6..

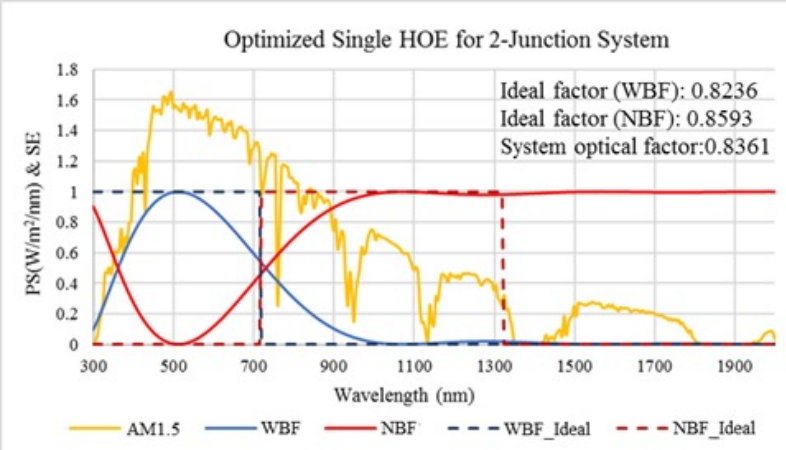

(a)

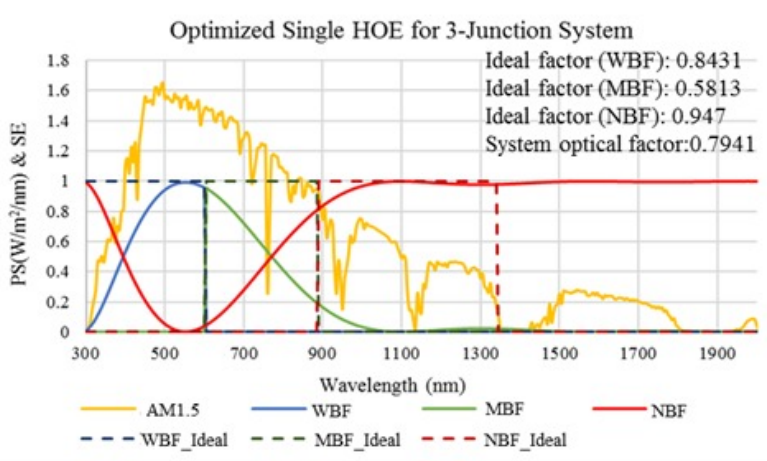

(c)

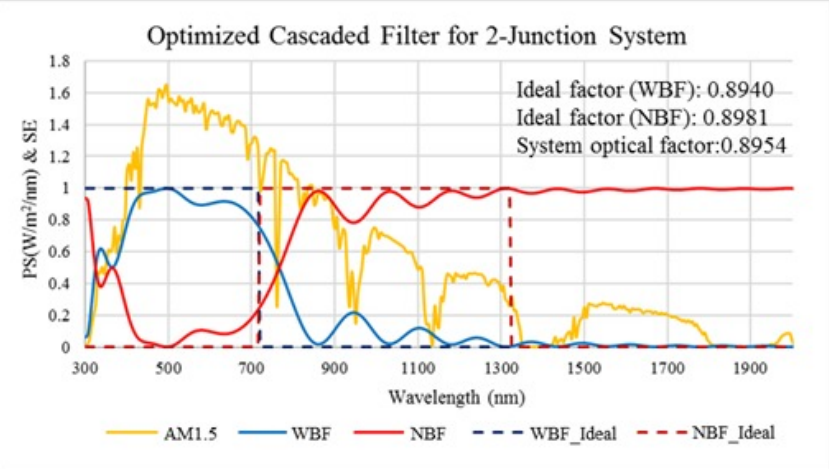

(b)

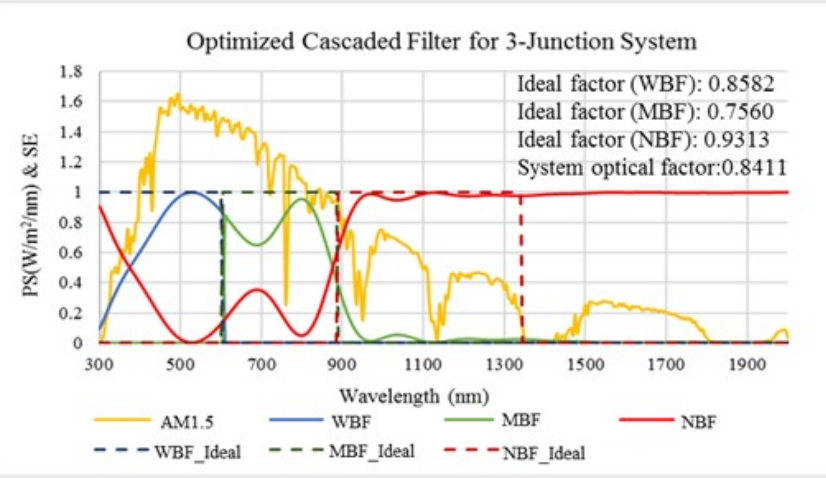

(d)

Figure 6. The spectral optical filtering efficiencies (SOFE) of all PV bandgaps using in optimized two- and three- junction SSPV systems along with the AM1.5 illumination power spectrum (PS). (a) and (b) represents the SOFEs of the optimized two-junction systems with using single HOE and cascaded filter. (c) and (d) represents the SOFEs of the optimized three-junction systems with using single HOE and cascaded filter. The red, blue and green curves are for the system filtering properties of the wide bandgap (WBF), the narrow bandgap (NBF) and the middle bandgap (MBF). 
The resulting filtering properties were then used to compute the power conversion efficiencies for the different configurations using the detailed balance method ${ }^{4}$. The improvement over best bandgap (IoBB) is also calculated for each system as a metric for system performance ${ }^{5}$. For comparison, the efficiencies of tandem MJ PV cells with the same bandgap combinations are calculated including the restriction due to current constraints. The results are summarized in Tables 4 . and 5. for the two- and three-junction bandgap systems.

With AM1.5 illumination the results show that the holographic SSPV systems described in this paper have the potential to reach a maximum power conversion efficiency of $42.56 \%$ using a DCG holographic filter in the two-junction system, and $48.41 \%$ for the three-junction system. The results also show an IoBB of a $37.73 \%$ for the two bandgap and $54.43 \%$ for the 3 band gap SSPV systems. In addition, the loss due to non-ideal DCG filter spectrum splitting is $7.60 \%$ for the 2 bandgap and $6.74 \%$ for the 3 bandgap SSPV systems.

Table 4. The power conversion efficiencies and IoBB for the optimized two-junction SSCPV systems, Tandem PV and ideal condition at AM1.5 solar irradiance.

\begin{tabular}{|l|c|c|c|c|c|}
\hline & \multirow{2}{*}{ Single BG } & \multicolumn{3}{|c|}{ Two-Junction (1.73eV; 0.94eV) } \\
\cline { 3 - 6 } & & Single HOE (SS) & Cascaded Filter (SS) & Tandem & Ideal \\
\hline Wide Bandgap (1.73eV) & $28.77 \%$ & $23.61 \%$ & $25.67 \%$ & $28.74 \%$ & $28.74 \%$ \\
\hline Narrow Bandgap (0.94eV) & $30.90 \%$ & $17.36 \%$ & $16.89 \%$ & $12.68 \%$ & $17.32 \%$ \\
\hline System Power Efficiency & & $\mathbf{4 0 . 9 7 \%}$ & $\mathbf{4 2 . 5 6 \%}$ & $\mathbf{4 1 . 4 2 \%}$ & $\mathbf{4 6 . 0 6 \%}$ \\
\hline IoBB & & $32.59 \%$ & $37.73 \%$ & $34.05 \%$ & $49.06 \%$ \\
\hline
\end{tabular}

Table 5. The power conversion efficiencies and IoBB for the optimized three-junction SSCPV systems, Tandem PV and ideal condition at AM1.5 solar irradiance.

\begin{tabular}{|l|c|c|c|c|c|}
\hline & \multirow{2}{*}{ Single BG } & \multicolumn{3}{|c|}{ Three-Junction (2.05eV; $1.4 \mathrm{eV} ; 0.93 \mathrm{eV})$} \\
\cline { 3 - 6 } & & Single HOE (SS) & Cascaded Filter (SS) & Tandem & Ideal \\
\hline Wide Bandgap (2.05eV) & $22.10 \%$ & $18.06 \%$ & $18.97 \%$ & $21.56 \%$ & $21.56 \%$ \\
\hline Middle Bandgap (1.4eV) & $33.24 \%$ & $11.33 \%$ & $14.88 \%$ & $13.26 \%$ & $19.67 \%$ \\
\hline Narrow Bandgap (0.93eV) & $30.70 \%$ & $16.38 \%$ & $14.56 \%$ & $7.50 \%$ & $10.68 \%$ \\
\hline System Power Efficiency & & $\mathbf{4 5 . 7 7 \%}$ & $\mathbf{4 8 . 4 1 \%}$ & $\mathbf{4 2 . 3 2 \%}$ & $\mathbf{5 1 . 9 1 \%}$ \\
\hline IoBB & & $49.09 \%$ & $54.43 \%$ & $27.3 \%$ & $56.17 \%$ \\
\hline
\end{tabular}

\section{CONCLUSIONS}

In this paper, a typical holographic dispersive spectrum splitting PV system with ideal concentration optics was demonstrated. The design can significantly improve the system power conversion efficiency of multiple bandgap PV systems with well-designed holographic filters. A systematic method for optimizing volume holographic elements for spectrum splitting systems was described. Single and cascaded holographic filters were modeled with approximate coupled wave analysis. A search algorithm evaluating hologram parameters was developed to optimize both single layer and two-layer cascaded holographic spectrum splitting elements for different bandgap combinations of two- and three-junction SSPV system illuminated with an AM1.5 solar spectrum. The variables that were searched in the optimization, were limited to the range available for DCG materials. Finally, the system power efficiencies were calculated with optimized filters using a detailed balance method. The results show that Under AM1.5 illumination, the holographic SSPV systems can reach a maximum limit of $42.56 \%$ and $48.41 \%$ of power conversion efficiency for two- and three-junction combination using two-layer cascaded holographic spectrum splitting elements. This results in an IoBB of $37.73 \%$ and a $54.43 \%$ and better performance than a tandem multi-junction cell with the same bandgap combinations.

\section{ACKNOWLEDGEMENTS}

The authors wish to acknowledge support from the NSF/DOE ERC cooperative agreement No. EEC-1041895, NSF grant ECCS-1405619. 


\section{REFERENCES}

[1] Polman, A. and Atwater, H. A., "Photonic design principles for ultrahigh-efficiency photovoltaics,” Nat. Mater. 11(3), 174-177 (2012).

[2] Mart'I, A. and Luque,A., [Next Generation Photovoltaics High efficiency through full spectrum utilization], Institute of Physics Publishing, Bristal \& Philadelphia, 108-119 (2004).

[3] McConnell R., “Concentrator Photovoltaic Technologies: Review Market Prospects,” Refocus, 6:35-9 (2005).

[4] Shockley, W., \& Queisser, H. J., "Detailed Balance Limit of Efficiency of p-n Junction Solar Cells,” Journal of Applied Physics, 32(3), 510-519 (1961).

[5] Russo, J. M., Zhang, D., Gordon, M., Vorndran, S. D., Wu, Y., and Kostuk, R. K., "Grating-over-lens concentrating photovoltaic spectrum splitting systems with volume holographic optical elements," Proc. SPIE 8821, 882106 (2013).

[6] Wu, Y. and Kostuk, R.K., "Two-junction holographic spectrum-splitting micro concentrating photovoltaic system. J. Photon. Energy. 0001;7(1):017001 (2017).

[7] Wu, Y., Vorndran, S., Pelaez, S. A. and Kostuk, R. K.," Three junction holographic micro-scale PV system ", Proc. SPIE 9937, Next Generation Technologies for Solar Energy Conversion VII, 99370M (2016).

[8] Vorndran, S. D., Chrysler, B., Wheelwright, B., Angel, R., Holman, Z., and Kostuk, K., "Off-axis holographic lens spectrum-splitting photovoltaic system for direct and diffuse solar energy conversion," Appl. Opt.55, 7522-7529 (2016).

[9] McCambridge, J. D., Steiner, M. A., Unger, B. L., Emery, K. A., Christensen, E. L., Wanlass, M. W., Gray, A. L., Takacs, L., Buelow, R., McCollum, T. A., Ashmead, J. W., Schmidt, G. R., Haas, A. W., Wilcox, J. R., Van Meter, J., Gray, J. L., Moore, D. T., Barnett, A. M. and Schwartz, R. J., "Compact spectrum splitting photovoltaic module with high efficiency," Prog Photovoltaics Res Appl 19(3), 352-360 (2011).

[10] Maragliano, C., Chiesa, M., and Stefancich, M., "Point-focus spectral splitting solar concentrator for multiple cells concentrating photovoltaic system,” J. Opt. 17(10), 105901 (2015).

[11] Broderick, L. Z., Albert, B. R., Pearson, B. S., Kimerling, L. C., Michel, J., "Design for energy: Modeling of spectrum, temperature and device structure dependences of solar cell energy production," Solar Energy Mater.Solar Cells 136, 48-63 (2015).

[12] Barnett, A., Kirkpatrick, D., Honsberg, C., Moore, D., Wanlass, M., Emery, K., Schwartz, R., Carlson, D., Bowden, S., et al., "Very high efficiency solar cell modules," Prog Photovoltaics Res Appl 17(1), 75-83 (2009).

[13] Ingersoll, G. B. and Leger J. R., "Optimization of multi-grating volume holographic spectrum splitters for photovoltaic applications," Appl. Opt. 55, 5399-5407 (2016).

[14] Chang, B. J. and Leonard, C. D., "Dichromated gelatin for the fabrication of holographic optical elements," Appl. Opt., 18(14), 2407-2417 (1979).

[15] Bremner, S. P., Levy, M. Y. and Honsberg, C. B., "Analysis of tandem solar cell efficiencies under AM1.5G spectrum using a rapid flux calculation method,” Prog. Photovolt: Res. Appl., 16: 225-233, (2008).

[16] Kogelnik, H.. “Coupled wave theory for thick hologram gratings,” Bell Syst. Tech. J., 48(9), 2909-2947 (1969). 